\title{
Increased preexcitation on electrocardiography improves accuracy of algorithms for accessory pathway localization in Wolff-Parkinson-White syndrome
}

\author{
Paweł Moskal'1, Adam Bednarski², Grzegorz Kiełbasa'1, Danuta Czarnecka'1, Marek Jastrzębski \\ 1 1st Department of Cardiology, Interventional Electrocardiology and Hypertension, Institute of Cardiology, Jagiellonian University Medical College, Kraków, Poland \\ 2 1st Department of Cardiology, Interventional Electrocardiology and Hypertension, University Hospital, Kraków, Poland
}

\section{KEY WORDS}

accessory pathway, algorithms, maximal preexcitation, preexcitation, Wolf-ParkinsonWhite syndrome
Correspondence to: Paweł Moskal, MD, 1st Department of Cardiology, Interventional Electrocardiology and Hypertension, Institute of Cardiology, Jagiellonian University Medical College, ul. Jakubowskiego 2, 30-688 Kraków, Poland, phone: +48124002150, email:pawel@moskal.in Received: April 16, 2020. Revision accepted: May 17, 2020. Published online: May 20, 2020. Kardiol Pol. 2020; 78 (6): 567-573 doi:10.33963/KP.15378 Copyright by the Author(s), 2020

\section{ABSTRACT}

BACKGROUND Several electrocardiographic (ECG) algorithms have been developed for predicting accessory pathway (AP) location in Wolff-Parkinson-White syndrome. However, their accuracy may be related to the manifested degree of preexcitation on ECG.

AIMS Our goal was to assess the effect of the degree of preexcitation on the accuracy of 4 traditional AP localization algorithms and to compare them with the algorithm specifically designed for ECGs with maximal preexcitation (Pambrun).

METHODS The study included 300 patients who underwent successful ablation of an overt atrioventricular AP. Resting and maximally preexcited ECGs obtained during incremental atrial pacing were assessed using 4 traditional AP localization algorithms: Xie, d'Avila, Iturralde, and Taguchi. Maximally preexcited ECGs were additionally assessed with the Pambrun algorithm. We compared the precision of the algorithms to predict accurate or anatomically adjacent AP location.

RESULTS The overall accuracy of traditional AP localization algorithms using resting ECG ranged between $26 \%$ and $53.7 \%$ and improved to a range of $47.3 \%$ to $69.7 \%$ when adjacent locations were accepted. When used with maximal preexcitation, all algorithms had significantly higher accuracy, with a mean improvement of 14.3 and 15.6 percentage points for precise and adjacent sites, respectively. The Pambrun algorithm for maximally preexcited ECGs had the highest precision for both accurate and adjacent locations of the APs ( $89.7 \%$ and $97 \%$, respectively).

CONCLUSIONS Greater preexcitation on ECG improved the accuracy of the traditional AP localization algorithms. The algorithm designed to use maximally preexcited ECGs has the best accuracy. Maximally preexcited ECG recordings should preferably be used in clinical practice to facilitate the ablation procedure.

INTRODUCTION Ventricular preexcitation caused by an accessory pathway (AP) is a typical feature of Wolff-Parkinson-White syndrome. The electrocardiographic (ECG) hallmark of preexcitation is the QRS complex that is distorted, prolonged, and often with altered polarity. The radiofrequency catheter ablation of an AP is a standard treatment in Wolff-ParkinsonWhite syndrome. ${ }^{1}$ Preprocedural prediction of the AP location facilitates the success of ablation and allows for preprocedural risk assessment. Over 20 different algorithms were created to predict the AP location based on the following resting 12-lead ECG features: delta wave morphology, QRS polarity, or the R/S amplitude ratio of QRS complexes. However, most of these algorithms had low accuracy in validation studies. ${ }^{2-6}$ This discrepancy might be 


\section{WHAT'S NEW?}

This study assessed the accuracy of 5 algorithms to predict accessory pathway location using resting and maximally preexcited 12-lead electrocardiogram (ECG). We found that the increased preexcitation improved the accuracy of all the algorithms. The Pambrun algorithm, based on maximally preexcited ECGs, was shown to have the best accuracy. Our main finding is that the use of maximally preexcited ECG recordings is preferable in clinical practice to facilitate the ablation procedure.

related to a different degree of preexcitation in the initial sets of ECGs and in the validation cohorts. The majority of the AP localization algorithms were designed to be used with resting
ECG, where QRS morphology results from a fusion of ventricular activation through the HisPurkinje conduction system and an AP. ${ }^{7}$ Fused QRS morphology is influenced mainly by 2 factors: 1) conduction via atrioventricular node, His bundle, and bundle branches, and 2) AP localization. However, during provoked or spontaneous maximal preexcitation, ventricular activation accomplished almost exclusively by the AP is observed, with the QRS morphology almost completely depending on the AP location (FIGURE 1). ${ }^{8}$

Variability in the degree of preexcitation in the QRS morphology can explain the difficulty
Resting ECG

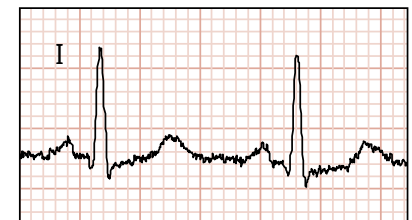

II

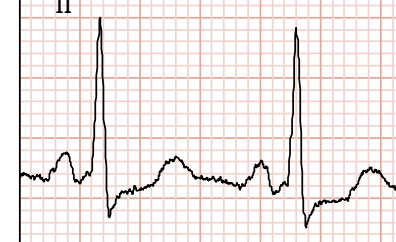

III

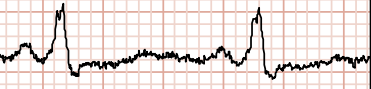

aVR

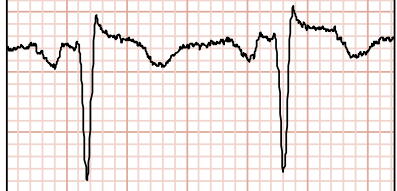

aVL

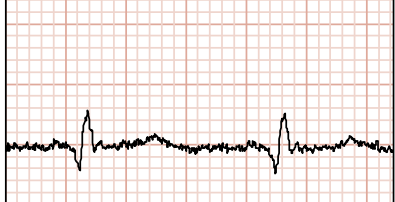

aVF

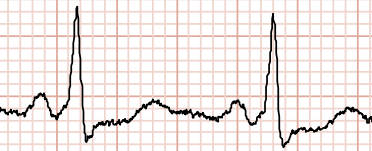

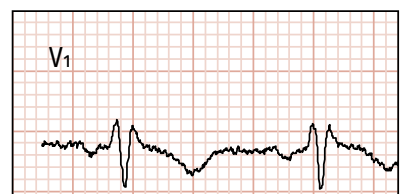

$V_{2}$

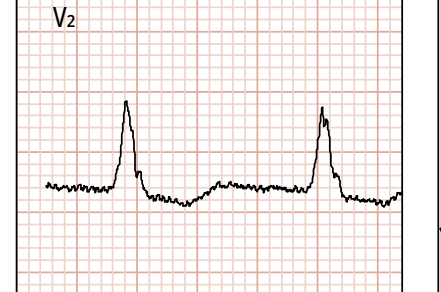

$V_{3}$
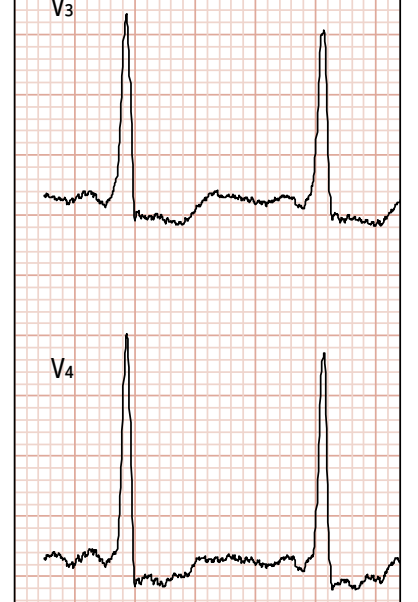

$V_{5}$

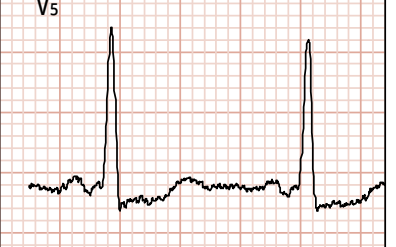

$V_{6}$

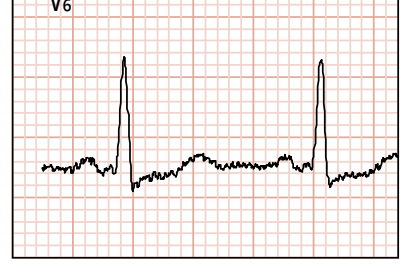

Maximally preexcited ECG
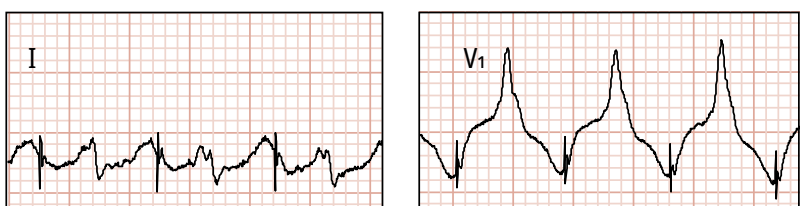

II
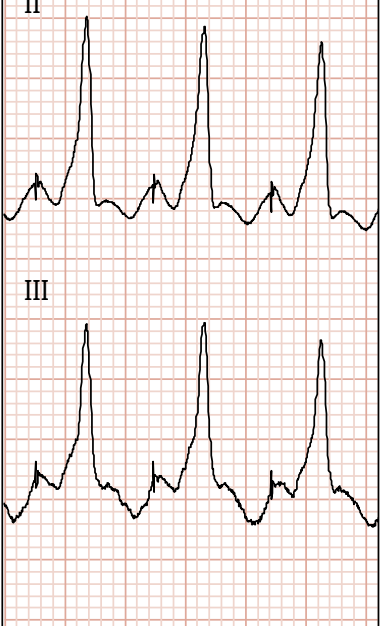

aVR

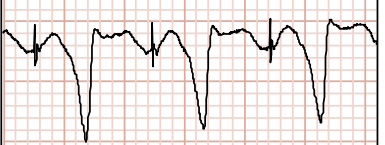

aVL
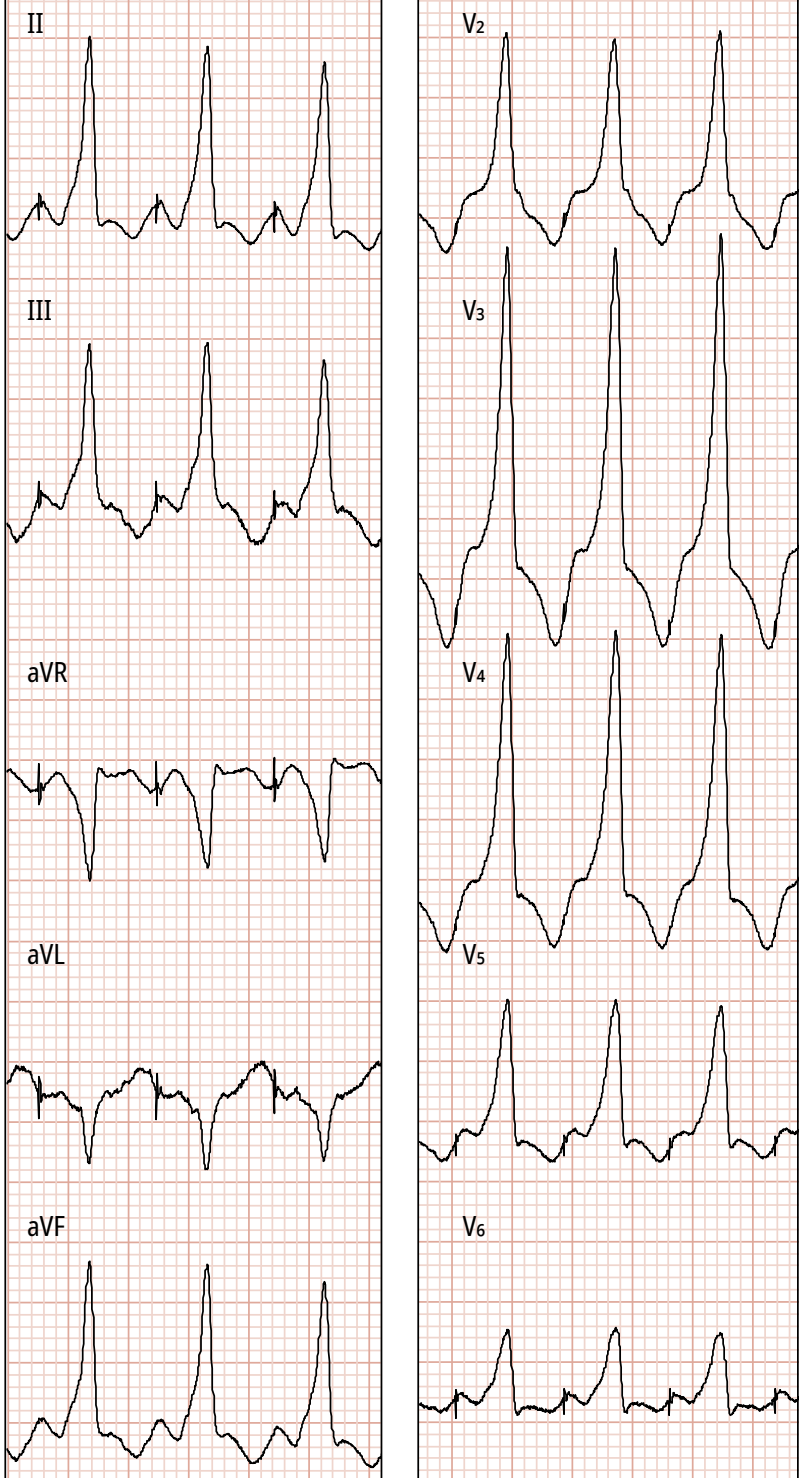

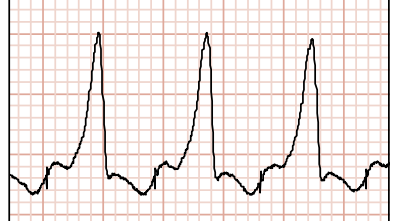

$V_{6}$

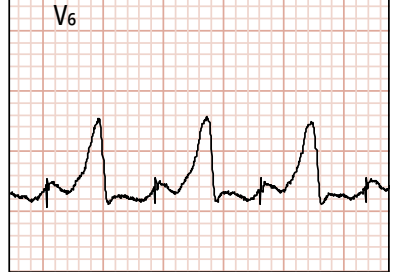

FIGURE 1 Examples of resting and maximally preexcited electrocardiograms (ECGs) recorded in a patient with a left lateral accessory pathway. The predictions of traditional algorithms were as follows: right lateral by Xie et al, ${ }_{12}$ anteroseptal by d'Avila et al, ${ }_{13}^{13}$ midseptal / posteroseptal by Taguchi et al, ${ }_{1}^{14}$ and right anterosuperior paraseptal by Iturralde et al. ${ }^{15}$ Using maximally preexcited ECG, Pambrun et al ${ }^{9}$ and all traditional algorithms correctly predicted the left lateral location. 
in creating an accurate and universal algorithm. Pambrun et $\mathrm{al}^{9}$ proposed a new AP localization algorithm designed for ECGs with maximal preexcitation that aims to have a better predictive value; however, it has not been validated by independent studies so far.

This study aimed to assess the effect of the degree of preexcitation on resting ECG on the accuracy of $4 \mathrm{AP}$ localization algorithms designed for resting ECGs, and to compare them to the recently introduced algorithm specifically designed for ECGs with maximal preexcitation.

METHODS We retrospectively analyzed 340 consecutive ablation procedures from 2002 to 2019 in patients with overt preexcitation. The ablation outcomes, success rates, and risk factors for malignant arrhythmias in this cohort were reported previously. ${ }^{10,11}$ For the current study, we excluded patients with structural heart disease, multiple APs, atriofascicular ("Mahaim") and fasciculoventricular pathways, failed ablation, and without high-quality 12-lead ECGs with resting and maximally preexcited patterns.

From the electrophysiological recording system, we printed a 12-lead surface ECG $(25 \mathrm{~mm} / \mathrm{s})$ with baseline preexcitation (observed at the beginning of the procedure). The second ECG recording was obtained during the electrophysiological study when maximal preexcitation was provoked by incremental fast atrial pacing, induction of antidromic antidromic atrioventricular reentry tachycardia, or induction of rapid atrial fibrillation. The AP location was assessed using the right and left anterior oblique views according to the position of the ablation catheter at the successful ablation site.
To compare the accuracy of AP prediction, we analyzed each pair of resting and maximally preexcited ECGs with 4 traditional algorithms developed by Xie et al, ${ }^{12}$ d'Avila et al, ${ }^{13}$ Iturralde et al,,$^{14}$ and Taguchi et $a l .{ }^{15}$ They were designed to use with resting 12-lead ECG and do not require subjective delta wave assessment. The Iturralde, d'Avila, and Xie algorithms predict the AP location at 5 to 9 sites along the mitral and tricuspid annulus, using mainly the polarity and morphology of the QRS complexes in leads II, III, $\mathrm{aVF}, \mathrm{aVL}, \mathrm{V}_{1}$, and $\mathrm{V}_{2}$. The Taguchi algorithm uses the $R / S$ ratio in leads $V_{1}, V_{2}$, and aVF to predict the location of the AP at 5 sites.

In addition, we assessed maximally preexcited ECGs with the recently published algorithm by Pambrun et al. ${ }^{9}$ To localize the AP at 9 sites, it uses the polarity of leads $\mathrm{V}_{1}$ and $\mathrm{V}_{3}$, the number of overall positive inferior leads (II, III, aVF), the ratio of $\mathrm{R}$ waves in leads $\mathrm{V}_{1}$ and lead $\mathrm{I}$, and the morphology of lead II. Algorithm criteria are summarized in Supplementary material, Table S1.

The number of possible AP locations in the selected algorithms ranges from 5 to 9 , and different terminology was used to describe them. To facilitate the comparison of the algorithms, we adapted a unified classification of AP locations, as it was done in previous studies. ${ }^{4,6}$ We used the AP sites reported by Pambrun et $\mathrm{al}^{9}$ as a reference and translated locations provided in other algorithms, as described in TABLE 1.

We defined the predictions of the algorithm as: 1) accurate (predictions matched precisely the AP location established during the electrophysiological study); 2) adjacent (predictions were anatomically adjacent to the actual AP location); and 3) inaccurate (predictions did not match the above definitions).

TABLE 1 Unified classification of accessory pathway nomenclature between algorithms

\begin{tabular}{|c|c|c|c|c|}
\hline \multirow{2}{*}{$\begin{array}{l}\text { AP location in the Pambrun algorithm, } \\
\text { reference }\end{array}$} & \multicolumn{4}{|c|}{ Algorithms } \\
\hline & Xie et al ${ }^{12}$ & d'Avila et a| ${ }^{13}$ & Iturralde et al ${ }^{14}$ & Taguchi et al ${ }^{15}$ \\
\hline Right anterior & RAS & AS & \multirow[t]{2}{*}{ RA } & \multirow[t]{2}{*}{ RAS/RA/RL } \\
\hline Right lateral & RL & RL & & \\
\hline Right posterior & $\mathrm{RP}$ & RPS & \multirow[t]{3}{*}{ RIP/RI } & $\mathrm{RPL} / \mathrm{RP}$ \\
\hline Right paraseptal & \multirow[t]{2}{*}{ RPS } & \multirow[t]{2}{*}{ PS } & & \multirow[t]{4}{*}{ MS/PS } \\
\hline Deep coronary sinus & & & & \\
\hline Nodo-Hisian & MS & MS & RASP & \\
\hline Left paraseptal & LPS & LPS & LIP / LI & \\
\hline Left posterolateral & LP/LPL & LP & \multirow[t]{2}{*}{ LPL/LAS } & LP/LPL \\
\hline Left lateral & LAL & LL & & $\mathrm{LA} / \mathrm{LL}$ \\
\hline
\end{tabular}

Abbreviations: AP, accessory pathway; AS, anteroseptal; DCS, deep coronary sinus; LA, left anterior; LAL, left anterolateral; LI, left inferior; LIP, left inferior paraseptal; LL, left lateral; LP, left posterior; LPL, left posterolateral; LPL, left posterolateral; LAS, left anterosuperior; LPS, left posteroseptal; MS, midseptal; PS, posteroseptal; RA, right anterior; RAS, right anteroseptal; RASP, right anterosuperior paraseptal; RI, right inferior; RIP, right inferior paraseptal; RL, right lateral; RP, right posterior; RPL, right posterolateral; RPS, right posteroseptal 
In the secondary analysis, we aimed to investigate the performance of traditional algorithms in patients divided according to the degree of preexcitation on resting ECG. We measured resting QRS duration (QRSd) with the global QRS method $^{16}$ and used terciles to classify ECGs into 3 groups according to the manifested preexcitation: 1) low, QRSd <123 ms ( $=109) ; 2)$ moderate, $\mathrm{QRSd}=123-138 \mathrm{~ms}(\mathrm{n}=92)$; and 3 ) high, QRSd $>138$ ms ( $\mathrm{n}=99)$.

The study protocol was approved by the local ethics committee. Patient consent was not required in this study.

Statistical analysis The accuracy of the algorithm was defined as the percentage of patients with a correct prediction of a successful ablation site. The $\mathrm{X}^{2}$ and McNemar tests were used to compare categorical variables and algorithm accuracy, respectively, between baseline and maximally preexcited ECGs. The Mann-Whitney test was used to compare the median QRS duration between left- and right-sided APs. A $P$ value of less than 0.05 was considered significant. The analysis was performed using STATA 16.1 (StataCorp, College Station, Texas, United States).

RESULTS The final study group included 300 patients. The demographic data and AP locations according to the successful ablation site during the electrophysiological study are presented in TABLE 2. Left free-wall APs were more prevalent than septal or right free-wall APs (44\%, $38 \%$, and $18 \%$, respectively). On resting ECG, the median QRS duration for left-sided APs was significantly shorter than for right-sided APs (127 ms vs $132 \mathrm{~ms} ; P=0.01$ ).

Accuracy of algorithms for resting and maximally preexcited electrocardiograms The overall accuracy of the algorithms is presented in TABLE3. For the ECGs with resting preexcitation, the Taguchi and Iturralde algorithms had the most accurate predictions $(53.7 \%$ and $53.3 \%$, respectively). When adjacent locations were accepted, the accuracy of all the algorithms improved, but the d'Avila, Iturralde, and Taguchi algorithms outperformed the Xie algorithm $(P<0.001)$.

For the ECGs with maximal preexcitation, all traditional algorithms showed an increase in accuracy, with a mean improvement of 14.3 percentage points for precise locations and 15.6 percentage points for adjacent locations. The Pambrun algorithm achieved the most accurate predictions for precise and adjacent sites.

Performance of algorithms according to the degree of preexcitation on resting electrocardiograms The accuracy of the algorithms in patients with low, moderate, and high resting preexcitation is presented in FIGURE2. The precision of all traditional algorithms improved with the increase of preexcitation, with a mean improvement from low to high resting preexcitation of 14.3 percentage points for accurate locations and 10 percentage points for adjacent sites. The Xie algorithm showed the lowest accuracy independently of the degree of preexcitation.

Accuracy in distinguishing between left- and right-sided accessory pathways The accuracy of the traditional algorithms in predicting

TABLE 2 Clinical characteristics of the study group $(n=300)$

Parameter

\begin{tabular}{|c|c|c|c|}
\hline \multicolumn{3}{|c|}{ Age, y, mean (SD) } & $32(18.4)$ \\
\hline \multicolumn{3}{|l|}{ Male sex } & $178(59.2)$ \\
\hline \multicolumn{3}{|c|}{ QRS duration on resting ECG, ms, mean (SD) } & $130.4(21)$ \\
\hline \multirow[t]{9}{*}{ AP location } & \multirow[t]{2}{*}{ Left free-wall } & Left lateral & $71(23.67)$ \\
\hline & & Left posterolateral & $61(20.33)$ \\
\hline & \multirow[t]{4}{*}{ Septal } & Left paraseptal & $17(5.67)$ \\
\hline & & Right paraseptal & $64(21.33)$ \\
\hline & & Parahisian / midseptal (nodo-Hisian) & $29(9.67)$ \\
\hline & & Epicardial (deep coronary sinus) & $4(1.33)$ \\
\hline & \multirow[t]{3}{*}{ Right free-wall } & Right anterior & $24(8)$ \\
\hline & & Right posterior & $19(6.33)$ \\
\hline & & Right lateral & $11(3.67)$ \\
\hline
\end{tabular}


TABLE 3 Overall accuracy of the accessory pathway localization algorithms on resting and maximally preexcited electrocardiograms

\begin{tabular}{|c|c|c|c|c|c|c|}
\hline \multirow[t]{2}{*}{ Algorithm } & \multicolumn{2}{|c|}{ Resting ECG } & \multicolumn{2}{|c|}{ ECG with maximal preexcitation } & \multicolumn{2}{|c|}{$P$ value (resting vs maximal preexcitation) } \\
\hline & Accuracy, \% & $\begin{array}{l}\text { Adjusted } \\
\text { accuracy }{ }^{2} \%\end{array}$ & Accuracy, $\%$ & $\begin{array}{l}\text { Adjusted } \\
\text { accuracya, \% }\end{array}$ & Accuracy & Adjusted accuracy \\
\hline Xie et al ${ }^{12}$ & 26 & 47.3 & 32.7 & 62.7 & $<0.001$ & $<0.001$ \\
\hline $\mathrm{d}^{\prime}$ Avila et al'13 & 40 & 64.3 & 54.7 & 79 & $<0.001$ & $<0.001$ \\
\hline Iturrlade et al ${ }^{14}$ & 53.3 & 69.7 & 67.7 & 86.3 & $<0.001$ & $<0.001$ \\
\hline Taguchi et al ${ }^{15}$ & 53.7 & 65.3 & 75 & 81 & $<0.001$ & $<0.001$ \\
\hline Pambrun et $a^{9}$ & - & - & 89.7 & 97 & - & - \\
\hline
\end{tabular}

a Predictions were anatomically adjacent to the actual AP location.

Abbreviations: ECG, electrocardiogram; others, see TABLE 1

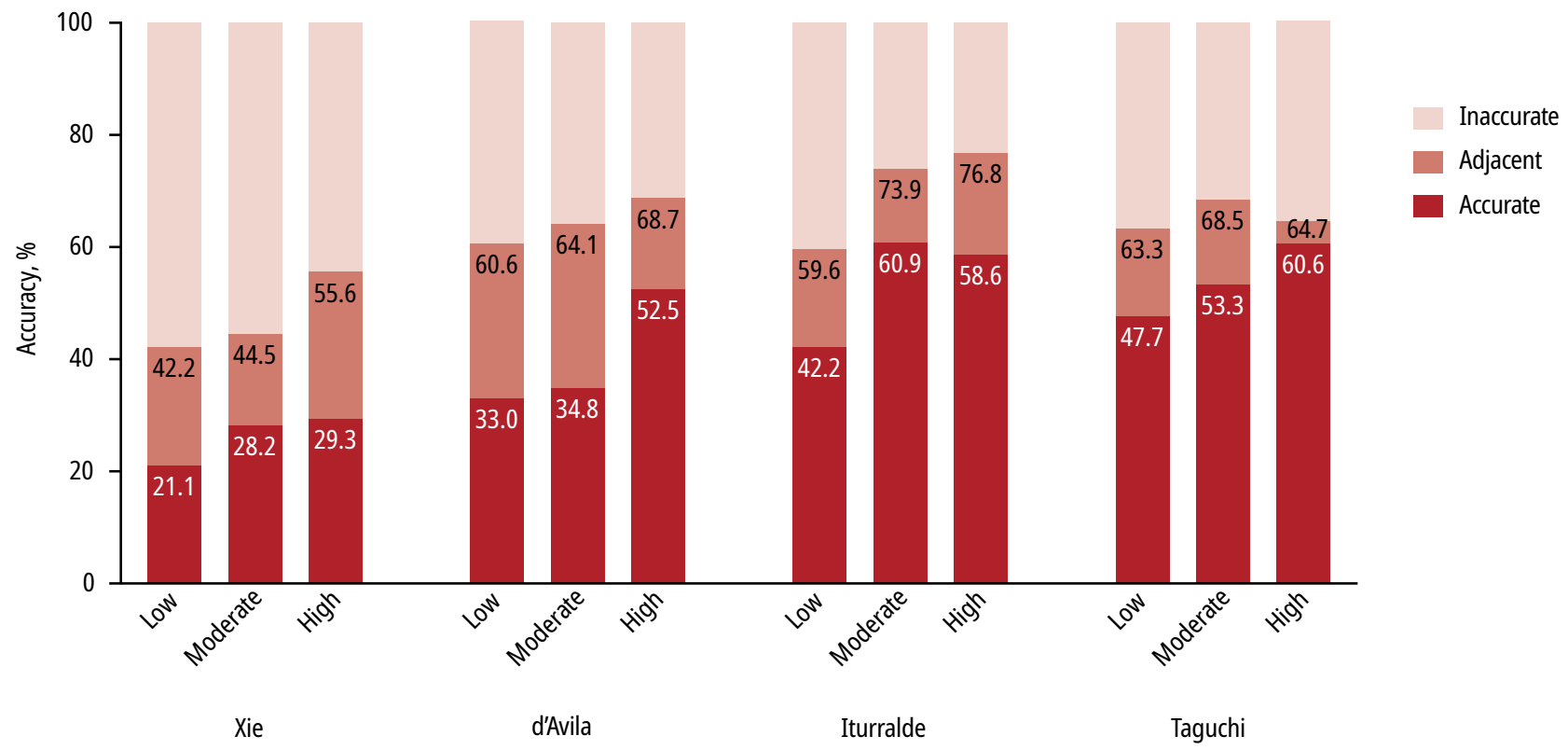

The degree of preexcitation

FIGURE 2 Accuracy of traditional algorithms according to the degree of manifested preexcitation on resting electrocardiogram (low, resting QRS duration [QRSd] <123 ms; moderate, QRSd = 123-138 ms; high, QRSd >138 ms)

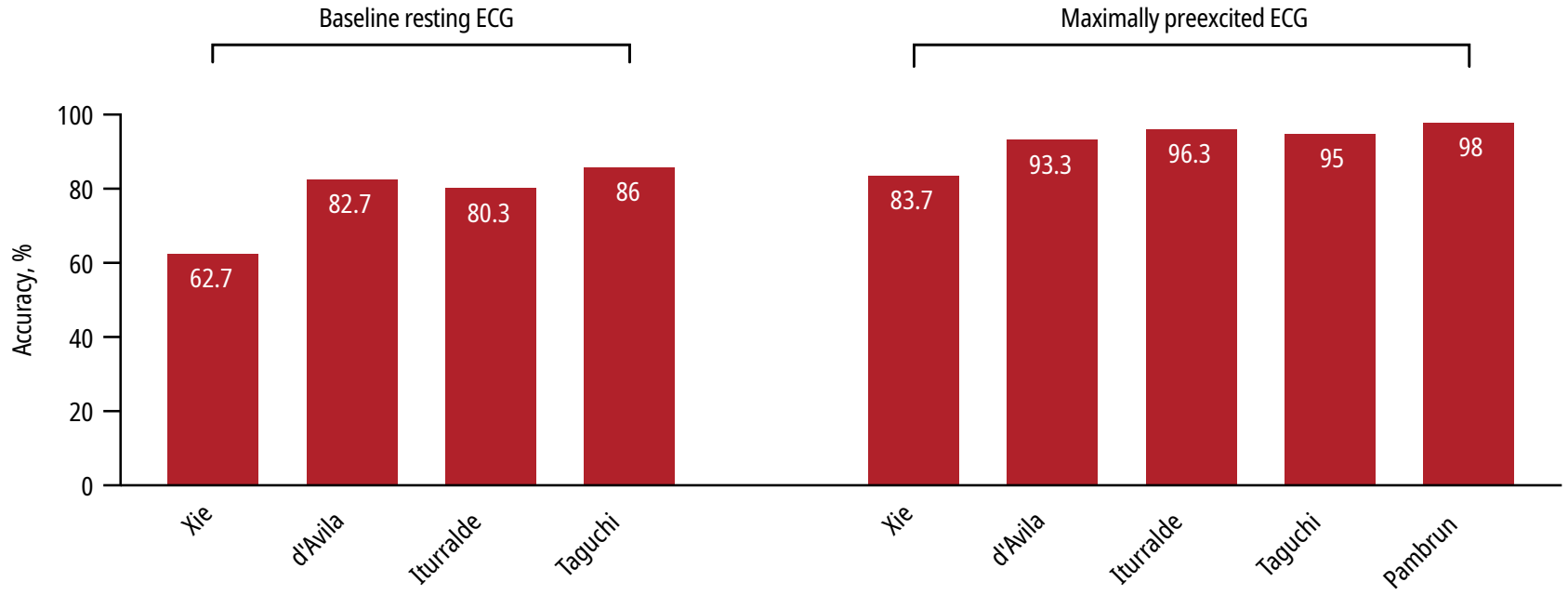

FIGURE 3 Accuracy of the algorithms to predict left- and right-sided accessory pathways using baseline and maximally preexcited electrocardiogram (ECG) 
left- and right-sided locations of APs using resting ECG ranged from $62.7 \%$ to $86 \%$ (FIGURE3). Using the maximally preexcited ECGs, the accuracy of the Xie, d'Avila, Iturralde, and Taguchi algorithms increased to a range of $83.7 \%$ to $95 \%$. The Pambrun algorithm was the most accurate (98\%).

Analysis of errors in the Pambrun algorithm In our cohort, $33 \%$ of parahisian APs manifested the overall negative polarity of the QRS complex in lead $\mathrm{V}_{3}$ during maximal preexcitation and were subsequently incorrectly predicted as a right anterior or right lateral AP. There were 4 epicardial or deep coronary sinus APs. None of them met the criteria of the Pambrun algorithm (Supplementary material, Table S1). In 3 cases, the QRS pattern was typical for right paraseptal AP. In one case, there were notched QS complexes in lead II. The fourth case had positive QRS complex in lead $V_{1}$ but did not have a notched QS complex in lead II.

DISCUSSION Our analysis of a large cohort of patients with Wolff-Parkinson-White syndrome showed that the accuracy of traditional algorithms in predicting AP location improved with the increase of preexcitation on resting ECG. Moreover, the traditional algorithms had better accuracy when applied to a maximally preexcited ECG obtained at the beginning of the electrophysiological study. In line with this, we found that the Pambrun algorithm, specifically designed for maximally preexcited ECGs, had the highest accuracy among all analyzed algorithms.

In our study, the Xie and Iturralde algorithms showed a similar accuracy in predicting AP locations as previous studies. ${ }^{2,5,6}$ Teixeira et $\mathrm{al}^{6}$ and Maden et $\mathrm{l}^{5}$ reported that the d'Avila algorithm was significantly worse than other algorithms, but in our research, its performance was comparable. The Taguchi algorithm has not been validated so far. We found that it was not better than the Iturralde algorithm, which is similarly designed to predict AP at 3 sites with only 3 criteria. The performance of all 4 traditional algorithms in discriminating a left-sided from right-sided AP was poor, with an error rate of $14 \%$ to $37.1 \%$.

It is generally assumed that the degree of preexcitation is associated with the accuracy of the AP localization algorithms, although this relationship has not been proved so far. Our study confirmed this clinical assumption. Low preexcitation on resting ECG hampered the predictive value of the traditional algorithms. On the other hand, the accuracy of the algorithms was significantly better when applied to ECGs with maximal preexcitation. For over 2 decades, the d'Avila algorithm has been routinely used in our electrophysiology laboratory, although with a modification in that we used fully preexcited ECG obtained at the beginning of the electrophysiological study instead of required resting ECG. This study confirms that it was the right approach, as the accuracy of this algorithm increased from $64 \%$ to $79 \%$. Similarly, about $15 \%$ more cases were correctly diagnosed by other algorithms when fully preexcited ECG was analyzed instead of ECG with baseline preexcitation. We believe that these results are firmly rooted in basic electrophysiology and ECG. A more preexcited QRS complex contains more information on the impact of an AP on ventricular depolarization. More information translates to a more accurate ECG analysis in terms of predicting the AP location around mitral and tricuspid annuli.

In the secondary analysis, we observed that the performance of the algorithms depended on the degree of preexcitation on resting ECG. This could be explained by the fact that the sensitivity of the algorithms varies between different $\mathrm{AP}$ locations, which, in turn, is the primary determinant of the degree of preexcitation. ${ }^{4-6}$ The algorithms have the worst ability to locate an AP at low preexcitation. Therefore, particular caution is needed when trying to predict the AP location in this group of patients.

Our study provides the first external validation of the Pambrun algorithm using a large cohort. We confirmed the high accuracy of the algorithm for precise localization of APs (89.7\%), which is in line with the accuracy reported by Pambrun et al (90\%). ${ }^{9}$ However, we found 2 recurring errors in nodo-Hisian (midseptal and parahisian area) and deep coronary sinus AP locations that need to be addressed. First, Pambrun et $\mathrm{al}^{9}$ reported that they did not have enough cases of AP localized in the coronary sinus diverticulum to fully assess the criterion for the deep coronary sinus location (notched QS complex in lead II). In our cohort, there were 4 epicardial APs, 3 of which were localized in the coronary sinus diverticulum. In all cases, the maximal preexcitation pattern was similar to the right paraseptal AP, with deep negative QS complexes in leads II, III, and aVF. Interestingly, in one of these cases, the criterion for deep coronary sinus location was present. We believe that this feature should also be assessed for the right paraseptal location to predict a location closer to the epicardium. Second, Pambrun et $\mathrm{al}^{9}$ used positive $\mathrm{QRS}$ complex in lead $\mathrm{V}_{3}$ as a criterion to differentiate the mid-septal and parahisian AP location (or nodo-Hisian using Pambrun's terminology) from the right lateral and right anterior AP. However, in our cohort, we found that almost $33 \%$ of true parahisian APs had overall negative $\mathrm{QRS}$ complex in lead $\mathrm{V}_{3}$ during maximal preexcitation; therefore, this feature cannot be reliably used to exclude this particular location.

Although the prediction of an AP location is better when based on maximally preexcited 
ECG, usually only resting ECG is available to clinicians. However, more preexcited ECG can be easily obtained, either using vagal maneuvers, short-acting drugs that slow conduction in the atrioventricular node, or by assessing 12-lead Holter monitoring, as the degree of preexcitation on ECG often changes during the day. During the electrophysiological study, maximally preexcited ECG should be obtained at the beginning of the procedure to verify resting ECG data.

Our study has several limitations. First, this was a single-center retrospective study. Despite a large number of patients, population-related differences could influence the accuracy of the tested algorithms. Second, resting ECGs were acquired at the beginning of the procedure, and the altered sympathetic/parasympathetic tone could affect the degree of baseline preexcitation.

In conclusion, our study confirms that the accuracy of traditional algorithms is related to the degree of preexcitation on resting ECG. Among all the investigated algorithms, the Pambrun algorithm, designed explicitly for maximally preexcited ECG, had the best accuracy for predicting AP location and should be preferably used for this purpose. When other algorithms are used, the fully preexcited ECG should be assessed to maximize their performance.

\section{SUPPLEMENTARY MATERIAL}

Supplementary material is available at www.mp.pl/kardiologiapolska.

\section{ARTICLE INFORMATION}

\section{CONFLICT OF INTEREST None declared.}

OPEN ACCESS This is an Open Access article distributed under the terms of the Creative Commons Attribution-Non Commercial-No Derivatives 4.0 International License (CC BY-NC-ND 4.0), allowing third parties to download articles and share them with others, provided the original work is properly cited not changed in any way, distributed under the same license, and used for noncommercial purposes only. For commercial use, please contact the journal office at kardiologiapolska@ptkardio.pl.

HOW TO CITE Moskal P, Bednarski A, Kiełbasa G, et al. Increased preexcitation on electrocardiography improves accuracy of algorithms for accessory pathway localization in Wolff-Parkinson-White syndrome. Kardiol Pol. 2020; 78: 567-573. doi:10.33963/KP.15378

\section{REFERENCES}

1 Giaccardi M. Ablation for Wolff-Parkinson-White syndrome: a life-saving procedure. Kardiol Pol. 2020; 78: 177-178.

2 Basiouny T, de Chillou C, Fareh S, et al. Accuracy and limitations of published algorithms using the twelve-lead electrocardiogram to localize overt atrioventricular accessory pathways. J Cardiovasc Electrophysiol. 1999; 10: 1340-1349.

3 Katsouras CS, Greakas GF, Goudevenos JA, et al. Localization of accessory pathways by the electrocardiogram: which is the degree of accordance of three algorithms in use? Pacing Clin Electrophysiol. 2004; 27: 189-193.

4 Wren C, Vogel M, Lord S, et al. Accuracy of algorithms to predict accessory pathway location in children with Wolff-Parkinson-White syndrome. Heart. 2012; 98: 202-206.

5 Maden 0, Balci KG, Selcuk MT, et al. Comparison of the accuracy of three algorithms in predicting accessory pathways among adult Wolff-Parkinson-White syndrome patients. J Interv Card Electrophysiol. 2015; 44: 213-219.

6 Teixeira CM, Pereira TA, Lebreiro AM, Carvalho SA. Accuracy of the electrocardiogram in localizing the accessory pathway in patients with Wolff-ParkinsonWhite pattern. Arq Bras Cardiol. 2016; 331-338.

7 Josephson ME. Preexcitation syndromes. In: Josephson's Clinical Cardiac Electrophysiology: Techniques and Interpretations. 5th ed. Wolters Kluwer; 2016: 336-440.
8 Jastrzębski M, Moskal P, Kukla P, et al. Specificity of wide QRS complex tachycardia criteria and algorithms in patients with ventricular preexcitation. Ann Noninvasive Electrocardiol. 2018; 23: e12493.

9 Pambrun T, El Bouazzaoui R, Combes N, et al. Maximal pre-excitation based algorithm for localization of manifest accessory pathways in adults. JACC Clin Electrophysiol. 2018; 4: 1052-1061.

10 Jastrzębski M, Moskal P, Pitak M, et al. Contemporary outcomes of catheter ablation of accessory pathways: complications and learning curve. Kardiol Pol. 2017; $75: 804-810$.

11 Moskal $P$, Jastrzębski M, Pitak M, et al. Malignant ventricular arrhythmias and other complications of untreated accessory pathways: analysis of prevalence and risk factors in over 600 ablation cases. Kardiol Pol. 2020; 78: 203-208.

12 Xie B, Heald SC, Bashir Y, et al. Localization of accessory pathways from the 12-lead electrocardiogram using a new algorithm. Am J Cardiol. 1994; 74: 161-165.

13 d'Avila A, Brugada J, Skeberis V, et al. A fast and reliable algorithm to localize accessory pathways based on the polarity of the QRS complex on the surface ECG during sinus rhythm. Pacing Clin Electrophysiol. 1995; 18: 1615-1627.

14 Iturralde P, Araya-Gomez V, Colin L, et al. A new ECG algorithm for the localization of accessory pathways using only the polarity of the QRS complex. J Electrocardiol. 1996; 29: 289-299.

15 Taguchi N, Yoshida N, Inden $\mathrm{Y}$, et al. A simple algorithm for localizing accessory pathways in patients with Wolff-Parkinson-White syndrome using only the R/S ratio. J Arrhythmia. 2014; 30: 439-443.

16 Jastrzebski M, Baranchuk A, Fijorek K, et al. Cardiac resynchronization therapy-induced acute shortening of QRS duration predicts long-term mortality only in patients with left bundle branch block. Europace. 2018; 21: 1-9. 